\title{
Marginación educativa y violencia: una intervención de lectoescritura en telebachillerato
}

\section{Educational marginalization and violence: A literacy intervention in a telebachillerato}

\author{
ESBEIDI YARET LARA REYES* \\ Antonia Olivia JaRvio Fernández ${ }^{* *}$
}

Este trabajo presenta un proyecto de intervención para promover la lectura y la escritura en un telebachillerato ubicado en una comunidad con marginación y violencia; la metodología usada fue la investigación-acción participativa. El propósito era suscitar el interés mediante la lectura estética y la escritura creativa con textos de literatura universal y sobre la violencia, además de propiciar la reflexión y el diálogo para dejar de normalizar prácticas de violencia y comprender el fenómeno para orientar acciones. El grupo constó de 24 jóvenes de entre 14 y 16 años. La evaluación se realizó con encuestas -una inicial y una final-, bitácoras de observación participante y ejercicios de lectura y escritura. Los resultados revelan un evidente progreso en las prácticas de lectura y escritura, así como en la reflexión y el diálogo para la desnaturalización de la violencia. Sin embargo, se reafirma que las condiciones precarias de la institución educativa constituyen un obstáculo.

This work presents an intervention to promote reading and writing in a high school located in a community with marginalization and violence. The participatory action research methodology was used. Interest was sought through aesthetic reading and creative writing, through universal literature texts and texts on violence. Likewise, promote reflection and dialogue to stop normalizing practices of violence and understand the phenomenon to guide actions. The group consisted of 24 young people between the ages of 14 and 16. The evaluation was carried out through surveys -an initial and a final one-, participant observation logs, as well as reading and writing exercises. The results reveal an evident progress in reading-writing practices, as well as in reflection and dialogue for the denaturalization of violence. However, it is reaffirmed that the precarious conditions in which the educational institution finds itself constitute an obstacle.

Recibido: 28 de enero de 2021 | Aceptado para publicarse: 5 de julio de 2021| Publicado: 24 de agosto de 2021

Cómo citar: Lara Reyes, E. Y. y Jarvio Fernández, A. O. (2021). Marginación educativa y violencia: una intervención de lectoescritura en telebachillerato. Sinéctica, Revista Electrónica de Educación, (57), e1288. https://doi.org/10.31391/S2007-7033(2021)0057-009

\footnotetext{
* Especialista en promoción de la lectura. Centro de Estudios de la Cultura y la Comunicación de la Universidad Veracruzana. Correo electrónico: esbeiditeray@gmail.com/ https://orcid.org/0000-0002-4876-1306

** Doctora en Metodología y Líneas de Investigación en Biblioteconomía y Documentación por la Universidad de Salamanca, España. Investigadora del Centro de Estudios de la Cultura y la Comunicación de la Universidad Veracruzana. Coordinadora de la Especialización en Promoción de la Lectura y del Programa Universitario de Formación de Lectores de la misma universidad. Líneas de investigación: profesionalización en promoción de la lectura, lectura digital, literacidad en la educación. Correo electrónico: ojarvio@uv.mx/ https://orcid.org/0000-0003-2350-0289
}

\section{Palabras clave:}

promoción de la lectura, lectura estética, escritura creativa, violencia, educación media superior

\section{Keywords:}

promotion of reading, aesthetic reading, creative writing, violence, high school education 


\section{INTRODUCCIÓN}

T eer y escribir son actividades imprescindibles para el buen desempeño escolar; su dominio requiere destrezas para la comprensión y el procesamiento de la información (Garrido, 2004; Cassany, 2006; Ramírez, 2019). La lectura y la escritura contribuyen a la apropiación del conocimiento y la obtención de aprendizajes significativos (Pérez, 2018). Su ejercicio favorece el desarrollo del lenguaje, la concentración, la memoria, el raciocinio, la personalidad, la sensibilidad y la intuición. Su importancia trasciende el ámbito académico, ya que permite contactar con el otro, participar en el mundo y construir maneras más reflexivas, profundas y humanas de ver la vida (Vázquez, 2003). "Mejorar la lectura nos muestra la diversidad del mundo y amplía nuestros horizontes. Mejorar la lectura nos ayuda a vivir mejor" (Garrido, 1998, p. 6).

Las prácticas de lectura y escritura son fundamentales en la educación media superior (EMS), ya que esta es la antesala de ingreso al nivel superior, en el que los jóvenes se enfrentan a procesos más complejos de aprendizaje. En el ámbito global, se establece que es en la EMS donde deben desarrollarse las habilidades y destrezas para localizar información, interpretar un texto y valorar su contenido, lo cual es imprescindible para una integración plena a un mundo en constante transformación (OCDE, 2006).

No obstante, son evidentes las dificultades académicas de estudiantes de bachillerato reportadas en diversos estudios (Grijalva, 2011; Weiss, 2012, 2018; Weiss et al., 2017) y pruebas nacionales e internacionales, los cuales las relacionan con problemas socioculturales, de planeación académica, baja calidad en los aprendizajes y escasa formación de los profesores. Es común el reporte de que en la práctica en el aula se evidencian importantes deficiencias en la comprensión de textos, así como en las limitaciones de los profesores para revertir el problema (García, Arévalo y Hernández, 2018).

De acuerdo con Weiss (2018), la escuela para los jóvenes en esta etapa adquiere un importante valor como espacio de socialización, lo que conduce a procesos de subjetivación. Por otro lado, terminar la EMS no solo significa el pase automático para continuar los estudios superiores y llegar a ser profesional, sino que también es la posibilidad de contar con cierto prestigio social, obtener un trabajo formal y, específicamente para las mujeres, puede implicar "superar la condición de género" (Weiss, 2018, p. 6).

En los últimos años, en México, se decidió garantizar la obligatoriedad para que los jóvenes cursaran la EMS, lo que implicó acciones dirigidas hacia la cobertura y la calidad de la educación en este nivel. De lo anterior surge el telebachillerato comunitario.

Por otra parte, los estudios sobre abandono escolar revelan que sus causas son multifactoriales: económicas, académicas y personales, aunque destacan las de naturaleza escolar, al no tener el gusto, interés o competencias para la escuela, de acuerdo con el Programa Nacional de Juventud 2014-2018 (Sedesol, 2014). En este sentido, la no comprensión de textos y el requerimiento de destrezas para el uso de nuevos soportes de búsqueda de información originan la baja intensidad en los estudios y, por lo tanto, influyen en la deserción. Por ello, la lectura se constituye en uno de los elementos indispensables para el aprendizaje, la elaboración de textos escritos y la comunicación

Lara / Jarvio. Marginación educativa y violencia: una intervención de lectoescritura en telebachillerato Sinéctica 57 www.sinectica.iteso.mx 
del conocimiento (Pérez, 2018). De ahí la importancia de potenciar estas habilidades y desarrollar las inteligencias del educando y la competencia enciclopédica (bagaje) para alcanzar niveles avanzados en inferencias e intertextualidades (Montealegre, 2011), necesarios para las actividades académicas posteriores.

A pesar de todos los esfuerzos hechos en México para mejorar la lectura y la escritura, se reportan bajos índices de desempeño en estos rubros. En el Programa Internacional para la Evaluación de Estudiantes 2018 se identificó que el 1\% se desempeñó en los niveles más altos de competencia, frente al 35\% que no obtuvo un nivel mínimo (OCDE, 2018). Asimismo, en el Plan Nacional para la Evaluación de los Aprendizajes (PLANEA), un tercio de los estudiantes se ubicó en el nivel más bajo y nueve de cada 100 en el nivel IV, el más alto (Instituto Nacional para la Evaluación de la Educación, 2017a). Además, tanto el PLANEA como la Evaluación de la Oferta Educativa registraron que el nivel socioeconómico fue un predictor del rendimiento educativo y que los resultados más desfavorables se presentaron en los telebachilleratos, los telebachilleratos comunitarios y los planteles pertenecientes al Colegio Nacional de Educación Profesional Técnico (Instituto Nacional para la Evaluación de la Educación, 2017b).

Sin duda, son muchos más los factores que intervienen para que el contexto mexicano sea desfavorable para el desarrollo de los jóvenes. A la mala calidad educativa y la consecuente deserción escolar, se agrega la insuficiencia de los presupuestos en universidades, lo que propicia la falta de oportunidades para que más jóvenes puedan integrarse a una carrera universitaria. Aunado a ello, los altos índices de pobreza, el desempleo y el consecuente desplazamiento territorial de miles de personas, el deterioro de las instancias de seguridad social, la creciente inseguridad, el demérito de la procuración de justicia, así como la corrupción gubernamental (Valenzuela, 2017), hacen que prevalezca un ambiente generalizado de vulnerabilidad que afecta en especial a los jóvenes.

Además del problema formativo de los jóvenes en México, hay otro de dimensiones sociales impactantes: el de la violencia, que se ha incrementado desde que el gobierno declaró la guerra contra el narcotráfico en 2006 (Rosen y Zepeda, 2015). Informes gubernamentales señalan que 2019 fue el año más violento en el último trienio (Secretariado Ejecutivo del Sistema Nacional de Seguridad Pública, 2020) y en 2020, pese a los casi cien mil elementos desplegados de la Guardia Nacional y al confinamiento por la pandemia, los homicidios se mantuvieron igual según cifras de este organismo. El nivel de paz retrocedió un 4.3\% y se consideró que la principal causa de muerte entre los jóvenes mexicanos era el homicidio (Institute for Economics \& Peace, 2018).

Por otro lado, en la Encuesta de Jóvenes en México se reportó que seis de cada diez jóvenes han estado expuestos a situaciones de violencia, y que cuatro de cada diez han experimentado o atestiguado violencia entre pares, es decir, entre amigos, maltrato en la escuela o lugar de trabajo, agresiones en las redes sociales o por teléfono. Destaca que gran parte de ellos la justifica, ya que no pueden identificarla (Observatorio de la Juventud en Iberoamérica, 2019).

Con la tipificación de estos problemas, decidimos realizar un proyecto de intervención con estudiantes que cursan la EMS en la modalidad de telebachillerato, que son centros que se instalan en comunidades rurales, zonas urbanas periféricas y semiurbanas, donde hasta hace poco no se ofrecía este nivel educativo. Este subsistema se 
basa en los programas de la Dirección General del Bachillerato; los profesores -de tres a seis por escuela- son solo facilitadores encargados de acompañar al estudiante, y deben mostrarse sensibles ante los códigos del contexto que los rodea. Para su labor, utilizan una guía didáctica y el video educativo, diseñados y actualizados, periódicamente, para las 43 materias que se imparten durante seis semestres.

Por medio de textos literarios que tratan el tema de la violencia, se buscó incentivar la expresión, la reflexión y el diálogo para desnaturalizar y comprender el fenómeno de la violencia, que se asume como una manifestación social sistematizada y estructural, la cual produce automatismo, invisibilidad e inercia en los sujetos. Esta problemática suele centrarse en la violencia moral y no en la física, ya que la primera se caracteriza por su legitimación a través de la costumbre -la cual preserva los sistemas de estatus que controlan la permanencia de las jerarquías en otros órdenes (racial, étnico, entre otros)-. Asimismo, utiliza mecanismos como la diseminación masiva o "naturalización", es decir, que se acepta como parte de los comportamientos normales, el arraigo a valores religiosos o familiares que la justifican, y el desconocimiento o falta de identificación de la conducta, lo que impide visualizarla y accionar contra ella; esto provoca que permanezca durante largos periodos (Segato, 2003).

El proyecto que aquí presentamos atendió a un grupo de 24 jóvenes de entre los 14 y 16 años de la comunidad rural Mahuixtlán, ubicada en el centro de Veracruz, México. Tiene un grado medio de marginación (Sedesol, 2010) y las actividades, en su mayoría, están relacionadas con la producción de caña. El trabajo de intervención se guio por el método de investigación-acción participativa que, de acuerdo con Sigalat et al. (2019), permite la integración de saberes, conocimientos y aprendizajes de los participantes para la acción transformadora.

\section{PARA ACERCAR A LOS JÓVENES A LEER Y A ESCRIBIR}

Diversas investigaciones señalan el valor de la motivación en el proceso lector (Guthrie, Wigfield \& Perencevich, 2004; Guthrie et al., 2006; Wigfield \& Wagner, 2005; Garbe, 2005; Gabbian y Orlando, 2018), sin dejar de considerar que la praxis de la lectura requiere contextos de apoyo (Garbe, 2005; Garrido, 2012). En la adolescencia se debe fortalecer el vínculo con los libros atendiendo gustos, necesidades, emociones, y utilizando expresiones culturales diversas, así como manifestaciones artísticas ligadas a la lectura. Dado el gran uso de los dispositivos electrónicos en esta etapa, es imprescindible su manejo para acercarlos a propuestas de lectura que les interesen y disfruten, como literatura de aventuras o de ciencia ficción. Proveerles de material de lectura, promover la asistencia a librerías y bibliotecas, acercarlos a actos relacionados con los libros... De acuerdo con las características de nuestro trabajo, consideramos utilizar la lectura estética y la escritura creativa a fin de suscitar un mayor interés.

La lectura estética es aquella que no es unidireccional, puesto que prevé el desarrollo o el fortalecimiento de las experiencias estéticas enfocadas en los sentidos y las sensibilidades; es decir, se apuesta por el pensamiento estético, basado en la creatividad y la imaginación, lo que posibilita la comprensión de fenómenos socioculturales (Rosenblat, 1978, citado en Pirela, 2019, pp. 35-37). Sin embargo, en el término subyace una complejidad mayor, ya que, además, considera "la capacidad 
para comprenderla en toda su extensión y el aprendizaje para entrenarla" (Morales, 2019 , p. 64). La postura relacionada con la sensibilidad, a riesgo de aparentar una simplificación, para los fines que se persiguen, no solo se justifica, sino que se recomienda.

Existen otros tipos de estrategias que coadyuvan a la interacción entre el texto y los lectores; una de estas es la lectura en voz alta, herramienta que ha mostrado su eficacia cuando se utiliza con grupos para promover la lectura, puesto que tiene como finalidad transmitir ideas o impresiones estéticas, así como fomentar la capacidad expresiva; actualmente, no solo se usa para perfeccionar habilidades lectoras, sino que su práctica y enseñanza pretende lograr apertura y cooperación en los escuchas (Jacinto-Roca, Landa y López, 2018).

La escritura puede funcionar como una herramienta psicológica que lleva al lector a obtener un razonamiento práctico-situacional, lo cual implica la organización del pensamiento y la elaboración de nuevos conocimientos. La escritura creativa coloca al sujeto como compositor y crea un vínculo íntimo entre su experiencia, conocimientos y el acto mismo; por ello, se relaciona con el mundo y valora el diálogo, el intercambio de ideas y las diferentes percepciones sobre un mismo fenómeno (Barrón, 2019). Ambas fueron seleccionadas para motivar intrínsecamente a los jóvenes y otorgarles un verdadero propósito comunicativo para que las perciban como algo esencial y significativo, ya que las prácticas académicas han sido insuficientes para evitar el desinterés de los jóvenes y el abandono de esas prácticas (Pontecorvo, 2002).

"Ahora sabemos que las imágenes públicas, reales o ficticias, influyen directamente en los comportamientos sociales" (Volpi, 2011, p. 120) y ante esto la lectura de literatura, con sus múltiples situaciones imaginadas, nos abre la posibilidad de reconocimiento de nosotros mismos y apreciar los sentimientos y temores, como los de los demás. Los textos literarios nos enfrentan al desciframiento de lo vivido y motivan nuestra capacidad de reflexionar e interpretar a través de los elementos denotativos y connotativos, lo que provoca una inmersión emocional del lector. Estas sensaciones se pueden traducir en experiencias que orienten comportamientos y juicios o valores (Yubero, Sánchez y Larrañaga, 2013).

\section{ОвJETIVO}

Nuestro objetivo es suscitar el interés por la lectura y la escritura mediante la lectura estética, la escritura creativa y otras manifestaciones artísticas para propiciar un cambio de actitudes y prejuicios relacionados con el estereotipo de ser lector a fin de que la lectura sea adoptada como una actividad cotidiana, personal y enriquecedora, además de incentivar la expresión, la reflexión y el diálogo para desnaturalizar y comprender el fenómeno de la violencia.

\section{Metodología}

Nuestro proyecto de intervención asume la metodología de investigación-acción participativa con la finalidad de comprender la realidad social y provocar ciertas transformaciones (Martínez, 2004; Sigalat et al., 2019). Para lograrlo, el proceso de investigación se realizó in situ, donde no solo se investigó, sino que se atendió el 
problema real. Al finalizar la intervención, se preguntó en qué grado se logró lo formulado para objetivar su acción, que alcanzará el fin cuando se haya aumentado o reducido algún elemento del problema inicial (Callejo y Viedma, 2006).

\section{Diseño y planeación de las estrategias}

\section{Contexto de la intervención}

El trabajo se diseñó en el marco del plan de estudios de la especialización en Promoción de la Lectura de la Universidad Veracruzana, revisión de literatura y de reportes de intervenciones similares. Los trabajos reportados persiguieron el objetivo de reflexionar sobre la violencia y sus prácticas normalizadas. Aunque en su mayoría no se centraban en la promoción de la lectura, sí recurrían a textos literarios para propiciar la introversión, discusión y el diálogo sobre una cultura de paz (Instituto de la Juventud, 2009; Aguilar-Caro, 2015; Fundación Mujeres, 2017; López, 2017; Loría-Canul, 2018).

El proyecto de intervención se realizó en el telebachillerato de Mahuixtlán en Coatepec, Veracruz, con el grupo de primer semestre, conformado por 24 jóvenes: nueve hombres y quince mujeres, de los cuales, durante la transición semestral, siete ya no se inscribieron; por este motivo, en febrero de 2020, solo ingresaron siete hombres y diez mujeres. En relación con las condiciones de infraestructura, el inmueble se encontraba inacabado. Cuentan con agua y luz, pero carecen de internet, laboratorio, biblioteca y cerco de seguridad. Además, constantemente se suspendían las clases, por lo que se incumplía el tiempo efectivo para la educación de los jóvenes. Los cursos eran acompañados por seis maestros y un coordinador, quienes mencionaron que a menudo escaseaban los materiales y las capacitaciones para algunas asignaturas, como Formación para el trabajo.

\section{Materiales de lectura}

La construcción de la cartografía literaria consideró las características del grupo (obtenidas con la aplicación de la primera encuesta), los objetivos y las estrategias del proyecto. Este instrumento se conformó de 18 obras literarias y seis con temas sobre violencia, música popular y clásica, así como un documental sobre la narcocultura en México. El material en copias de los textos se entregó a los jóvenes para ser leídos durante las sesiones; se utilizaron fragmentos de obras, cuentos, relatos o poemas cortos, y se habilitó el préstamo de libros para los interesados que quisieran leer las versiones completas.

Instrumentos de evaluación

La batería de instrumentos de evaluación estuvo compuesta por:

- Las evaluaciones diagnóstica inicial y final, formadas por ítems cualitativos y cuantitativos. Estas consideraron la valoración del gusto, el interés y los hábitos de lectura, así como hábitos, intereses y ocupaciones personales, además 
de concepciones acerca de la violencia y del ser lector. La evaluación final integró un apartado para opiniones, valoraciones, sugerencias y recomendaciones acerca de la intervención.

- La bitácora registró lo ocurrido en las sesiones sobre las lecturas utilizadas, estrategias aplicadas, nivel de participación o disponibilidad del grupo, contingencias y observaciones, así como la fecha, el número de asistentes y el número de sesión.

-Para la evidencia de ejercicios se integraron fotografías del grupo y de actividades sobresalientes previa solicitud de permisos, debido a que se trabajó con menores de edad.

- Los resultados se reportaron con análisis descriptivos y gráficos a partir de las evidencias cuantitativas y cualitativas.

Implementación del proyecto de intervención

La intervención se desarrolló de noviembre de 2019 a marzo de 2020, y comprendió 18 sesiones presenciales, las cuales fueron realizadas en el salón de clases. Por lo general, al iniciar la sesión se leía en voz alta el texto correspondiente. Después, se efectuaba una actividad relacionada con lo leído; al final, se compartía el resultado del ejercicio. Cada dos sesiones de textos de literatura -en los que predominaba la literatura universal-, se intercalaba uno relativo a la violencia a fin de no afligir a los jóvenes con las otras lecturas; siempre se alentó a la reflexión y discusión sobre los temas tratados.

Las actividades se enfocaron a relacionar al lector con el texto; por ejemplo, con la lectura de El país de las metrallas, se hizo la lectura hasta un punto marcado en las copias. Los participantes debían escribir cómo creían que terminaba la historia -utilizando su creatividad e imaginación-. Después, se leyeron los escritos a los asistentes. Para que comprendieran el fenómeno sociocultural, explicamos los tipos de violencia identificados en la lectura y les pedimos que escribieran una reflexión sobre el tema.

\section{Estrategias de lectura en voz alta}

La lectura en voz alta colectiva generó interés en el grupo y sirvió para desinhibirlos, para que confrontaran las entonaciones, respetaran las pausas de los signos de puntuación, y escucharan atenta y participativamente. También, funcionó como estrategia de manejo y control del grupo, debido a que permanecían atentos para no obstruir la lectura mientras no tocara su turno. De igual modo, se realizó la lectura en voz alta por parte de la interventora.

\section{Estrategias de escritura creativa}

Organizamos sesiones de escritura creativa "Escribiendo cuentos I" y "Escribiendo cuentos II", con actividades como inventar el final del cuento, escritura de poemas o relatos cortos, y la formulación de preguntas con respuestas poéticas. Funcionaron todas 
las estrategias de escritura, ya que los jóvenes no solo escribieron los textos solicitados, sino que llegaron a elaborar cuentos y poemas complejos, enriquecidos con su creatividad, imaginación y en los que percibimos la influencia de la literatura comentada.

Estrategias de imaginación y creatividad

Realizamos estrategias de promoción de la lectura como de papiroflexia; el juego de la lechuga-pelota, que consiste en escribir preguntas (por ejemplo, ¿qué es la luna?) en hojas enrolladas como las de una lechuga, en las que les solicitamos una respuesta literaria y no literal; la creación oral colectiva de personajes con características extraordinarias y la complementación creativa de textos a partir de un formato fijo, con lo que incentivamos la imaginación al particularizarlas con su ingenio. La misma actividad dio resultados diferentes dadas las características de la creatividad, imaginación y experiencia de cada uno de los jóvenes.

Acercamiento a actividades artísticas relacionadas con la lectura-escritura

Recurrimos a canciones populares, como Las batallas y El niño yuntero, las cuales estuvieron relacionadas con las lecturas. Los jóvenes pudieron identificar que la diégesis de los textos leídos no solo se encontraba en los libros, sino en la radio como parte de su cotidianidad. Les presentamos la Three Gymnopédies: I: Lent et douloureux, del compositor Erik Satie, pieza que motivó su concentración durante la sesión "Escribiendo cuentos II". Finalmente, proyectamos el documental Narco cultura con la finalidad de reflexionar y discutir.

Definición sobre el significado de ser lector

A partir de los resultados de la encuesta diagnóstica, se estableció que ser lector es ser aplicado, interesante o sabio, que tiene ideas y puede expresar lo que siente; es decir, el concepto está relacionado con el buen desempeño de las actividades escolares, lo que implica obligación, esfuerzo y dificultad. No se concibe como una actividad que puede integrarse como disfrutable y realizarse por elección propia. Con las lecturas y actividades a lo largo del taller se buscó cambiar este concepto.

Expresión, reflexión y diálogo para la desnaturalización y comprensión de la violencia

Planteamos reglas de expresión sobre la violencia: tolerancia y respeto; omitir o cambiar los nombres reales implicados dentro de las reflexiones o comentarios emitidos; mantener una alta participación. La lectura Balas de salva sirvió para explicar la diferencia entre la realidad y la ficcionalización de la realidad. Para la caracterización e identificación de los tipos de violencia, su diálogo y reflexión, utilizamos los Poemas de terror y de misterio, El país de las metrallas, Insensatez, Estrella de la calle sexta y Narco cultura.

Las estrategias implementadas fueron la reflexión oral y escrita; el juego de la lechuga-reflexiva; el diálogo con preguntas como ¿qué tipo de violencia se encuentra en 
el texto?, ¿qué opinas sobre las acciones de tal personaje?, ¿qué factores del contexto crees que influyeron para que ocurriera eso? y ¿qué ideología existe detrás del discurso? Los jóvenes escuchaban y después emitían opiniones. Finalmente, reflexionaron y dialogaron sobre un documental relativo a la violencia.

Tabla 1. Descripción de las actividades por sesión

\begin{tabular}{|c|c|c|}
\hline $\begin{array}{l}\text { Información de } \\
\text { sesión }\end{array}$ & Lecturas & Desarrollo \\
\hline $\begin{array}{l}\text { Sesión } 1 \\
\text { Asistentes: } 24 \\
\text { Fecha: } \\
\text { 13/11/2019 }\end{array}$ & & $\begin{array}{l}\text { Presentación formal de los objetivos y formas de } \\
\text { trabajo entre la tallerista y los asistentes. Aplicación de } \\
\text { encuesta diagnóstica }\end{array}$ \\
\hline $\begin{array}{c}\text { Sesión } 2 \\
\text { Asistentes: } 23 \\
\text { Fecha: } \\
\text { 19/11/2019 }\end{array}$ & $\begin{array}{l}\text { Arreola, J. J., y Cuevas, J. L. (2002). } \\
\text { Confabulario. Fondo de Cultura } \\
\text { Económica ["El rinoceronte”, "La } \\
\text { jirafa”, "El hipopótamo", “Baby } \\
\text { HP”] }\end{array}$ & $\begin{array}{l}\text { Lectura en voz alta individual de los textos selecciona- } \\
\text { dos. La Act.1 consistió en escritura creativa. Se imaginó } \\
\text { y escribió un relato similar a los de "Bestiario" } \\
\text { En la Act. } 2 \text { todos imaginaron e inventaron, incluida la } \\
\text { tallerista, un personaje parecido a Baby HP -un híbrido } \\
\text { entre naturaleza y tecnología-; se indicó que se inten- } \\
\text { tara darle un propósito útil a sus características }\end{array}$ \\
\hline $\begin{array}{l}\text { Sesión } 3 \\
\text { Asistentes: } 23 \\
\text { Fecha: } \\
\text { 25/11/2019 }\end{array}$ & $\begin{array}{l}\text { Lemus, R. (2006). Balas de Salva. } \\
\text { Letras Libres, núm. 81, pp. 39-42 } \\
\text { Fabre, L. F. (2012). Poemas de } \\
\text { terror y de misterio. Almadía } \\
\text { [“Tráiler 1" “Dicen”, “3”] }\end{array}$ & $\begin{array}{l}\text { Lectura en voz alta colectiva de los textos. La Act.1 con- } \\
\text { sistió en la escritura creativa acerca de la violencia a } \\
\text { partir de un monstruo que compartiera características } \\
\text { con algún personaje violento. La interventora introdu- } \\
\text { jo el tema de la violencia y mencionó las reglas para } \\
\text { conversar sobre el tema. Finalmente, se leyó en voz alta } \\
\text { colectiva partes del texto. Se realizaron comentarios } \\
\text { y reflexiones acerca de la frontera entre la ficción y la } \\
\text { realidad }\end{array}$ \\
\hline $\begin{array}{l}\text { Sesión } 4 \\
\text { Asistentes: } 22 \\
\text { Fecha: } \\
\text { 26/11/2019 }\end{array}$ & $\begin{array}{l}\text { Benedetti, M. (2014). El amor, las } \\
\text { mujeres y la vida. Alfaguara ["Ha- } \\
\text { gamos un trato", "No te salves", } \\
\text { "Viceversa"] } \\
\text { Galeano, E. (2010). El fútbol a sol } \\
\text { y sombra (2010). Siglo XXI ["El } \\
\text { hincha", "El arquero", "El ídolo"] }\end{array}$ & $\begin{array}{l}\text { Lectura en voz alta individual de poemas de Mario } \\
\text { Benedetti. Lectura en voz alta colectiva de textos de } \\
\text { Eduardo Galeano. La Act.1 consistió en la escritura } \\
\text { creativa de un poema corto acerca del amor o del futbol } \\
\text { para, posteriormente, leer algunos de los escritos en } \\
\text { voz alta }\end{array}$ \\
\hline $\begin{array}{l}\text { Sesión } 5 \\
\text { Asistentes: } 21 \\
\text { Fecha: } \\
\text { 27/11/2019 }\end{array}$ & $\begin{array}{l}\text { Benedetti, M. (2014). El amor, las } \\
\text { mujeres y la vida. Alfaguara ["In- } \\
\text { timidad", "Ustedes y nosotros", } \\
\text { "Táctica y estrategia"] } \\
\text { Galeano, E. (2010). El fútbol a sol } \\
\text { y sombra (2010). Siglo XXI ["El } \\
\text { futbol", "El fanático", "El gol”] }\end{array}$ & $\begin{array}{l}\text { Lectura en voz alta individual de los poemas de Be- } \\
\text { nedetti. Lectura en voz alta colectiva de los textos de } \\
\text { Galeano. La Act.1 consistió en el juego de la lechuga- } \\
\text { pelota y en escribir una pregunta en cada hoja. Se hace } \\
\text { bolita la primera y las demás se ponen como capas } \\
\text { formando una pelota. Se lanza de una persona a otra y } \\
\text { esta debe responder la pregunta que le tocó }\end{array}$ \\
\hline $\begin{array}{c}\text { Sesión } 6 \\
\text { Asistentes: } 20 \\
\text { Fecha: } \\
\text { 02/12/2019 }\end{array}$ & $\begin{array}{l}\text { Rodríguez, F. (2013). El país de } \\
\text { las metrallas (pp. 54-57). Progra- } \\
\text { ma Cultural Tierra Adentro }\end{array}$ & $\begin{array}{l}\text { Lectura en voz alta individual interrumpida para reali- } \\
\text { zar la Act.1. consistente en que los asistentes escriban } \\
\text { su final de la historia; después, se lee en voz alta el } \\
\text { final original escrito por el autor. Finalmente, se leen } \\
\text { algunos finales alternos. En la Act.2 se escribió una } \\
\text { reflexión acerca de la violencia y los tipos de violencia } \\
\text { localizados en el texto }\end{array}$ \\
\hline $\begin{array}{c}\text { Sesión } 7 \\
\text { Asistentes: } 21 \\
\text { Fecha: } \\
\text { 10/12/2019 }\end{array}$ & $\begin{array}{c}\text { Agustín, J. (2014). La tumba (pp. } \\
\text { 98-106). Debolsillo }\end{array}$ & $\begin{array}{c}\text { Lectura en voz alta colectiva del fragmento de José } \\
\text { Agustín. Se propuso el "Taller de escritura creativa I", } \\
\text { por lo que redactaron un cuento de alguna experiencia } \\
\text { real o ficticia de su adolescencia }\end{array}$ \\
\hline
\end{tabular}




\begin{tabular}{|c|c|c|}
\hline $\begin{array}{l}\text { Sesión } 8 \\
\text { Asistentes: } 21 \\
\text { Fecha: } \\
\text { 11/12/2019 }\end{array}$ & $\begin{array}{l}\text { Al Berto (2016). Tres cartas de } \\
\text { la memoria de las Indias (pp. 20- } \\
\text { 23). Valparaíso México } \\
\text { Tovar, L. (2008). Diccionario del } \\
\text { mar. Universidad Veracruzana }\end{array}$ & $\begin{array}{c}\text { Lectura en voz alta individual de los poemas de Al } \\
\text { Berto. Después, los jóvenes decían una palabra relacio- } \\
\text { nada con el mar y se buscaba en el Diccionario del Mar. } \\
\text { Posteriormente, era leída en voz alta. La Act.1 consistió } \\
\text { en realizar un barco de papiroflexia. Nombraron a su } \\
\text { barco en relación con el texto leído. Al final, se leyeron } \\
\text { los nombres de los barcos y se explicó la razón del } \\
\text { nombre }\end{array}$ \\
\hline $\begin{array}{l}\text { Sesión } 9 \\
\text { Asistentes: } 20 \\
\text { Fecha: } \\
\text { 16/12/2019 }\end{array}$ & $\begin{array}{l}\text { Moya-Castellanos, H. (2005). } \\
\text { Insensatez (pp.28-33). Tusquets } \\
\text { Editores }\end{array}$ & $\begin{array}{l}\text { Lectura en voz alta colectiva del texto de Castellanos- } \\
\text { Moya. Se formularon comentarios orales sobre la } \\
\text { lectura. Finalmente, se realizó el juego de la lechuga } \\
\text { con preguntas reflexivas acerca de la violencia: ¿qué es } \\
\text { la discriminación?, ¿qué es la solidaridad?, ¿por qué es } \\
\text { mejor entablar un diálogo en vez de pelear?, etcétera }\end{array}$ \\
\hline $\begin{array}{l}\text { Sesión } 10 \\
\text { Asistentes: } 21 \\
\text { Fecha: } \\
\text { 13/01/2020 }\end{array}$ & $\begin{array}{l}\text { Huerta, E. (2014). Poesía com- } \\
\text { pleta (pp.154-157). Fondo de } \\
\text { Cultura Económica }\end{array}$ & $\begin{array}{l}\text { Lectura en voz alta colectiva de los poemínimos. La } \\
\text { Act.1 consistió en un ejercicio creativo de escribir } \\
\text { uno o dos poemínimos con tema libre. Al finalizar, se } \\
\text { comentó la estructura y composición de un poemínimo. } \\
\text { Para cerrar, algunos asistentes leyeron en voz alta sus } \\
\text { escritos }\end{array}$ \\
\hline $\begin{array}{l}\text { Sesión } 11 \\
\text { Asistentes: } 22 \\
\text { Fecha: } \\
\text { 15/01/2020 }\end{array}$ & $\begin{array}{l}\text { Quiroga, H. (2011). La gallina } \\
\text { degollada. NoBooks Editorial }\end{array}$ & $\begin{array}{l}\text { Lectura en voz alta colectiva del cuento de Quiroga. La } \\
\text { Act.1 consistió en un ejercicio creativo. Se repartieron } \\
\text { papelitos en blanco para que escribieran una pregunta } \\
\text { (por ejemplo: ¿qué es la felicidad?). Se recogieron los } \\
\text { papelitos y se volvieron a repartir. Otra persona los } \\
\text { respondió buscando evadir la literalidad y llevar un } \\
\text { proceso poético. Finalmente, las respuestas se leyeron } \\
\text { en voz alta }\end{array}$ \\
\hline $\begin{array}{l}\text { Sesión } 12 \\
\text { Asistentes: } 20 \\
\text { Fecha: } \\
\text { 10/02/2020 }\end{array}$ & $\begin{array}{l}\text { Crosthwaite, L. H. (2016). Estrella } \\
\text { de la calle sexta. Tusquets }\end{array}$ & $\begin{array}{l}\text { Lectura en voz alta individual de fragmentos de la } \\
\text { novela de Crosthwaite. Al finalizarla, se tornó el diálogo } \\
\text { hacia la reflexión y se comentaron los tipos de violen- } \\
\text { cia identificados. La Act.1 consistió en completar un } \\
\text { formato llamado “Mi palabra es..., diseñado y enfocado } \\
\text { para guiar la reflexión acerca de la violencia. Cada uno } \\
\text { leyó lo que escribió y, al final, comentó la impresión } \\
\text { que le dejaron los escritos creados }\end{array}$ \\
\hline $\begin{array}{l}\text { Sesión } 13 \\
\text { Asistentes: } 17 \\
\text { Fecha: } \\
\text { 17/02/2020 }\end{array}$ & $\begin{array}{l}\text { Pacheco, J. E. (2001). Las batallas } \\
\text { en el desierto. Era. (pp. 27-35 y } \\
\text { pp.35-40) } \\
\text { Café Tacvba (1992). Las batallas } \\
\text { [Canción]. En Café Tacvba, War- } \\
\text { ner Music México }\end{array}$ & $\begin{array}{l}\text { Lectura en voz alta colectiva de los primeros dos capí- } \\
\text { tulos seleccionados de Pacheco. En la Act.1 escribieron } \\
\text { cómo creen que se desarrolla la historia. Se reprodujo } \\
\text { la canción Las Batallas y se comentó el desenlace de la } \\
\text { novela, ya que pudo inferirse a través de la canción }\end{array}$ \\
\hline $\begin{array}{l}\text { Sesión } 14 \\
\text { Asistentes: } 17 \\
\text { Fecha: } \\
\text { 18/02/2020 }\end{array}$ & $\begin{array}{l}\text { Maldonado, M. (2016). El libro } \\
\text { de los oficios tristes. Comalcalco: } \\
\text { Ediciones Monte Carmelo } \\
\text { Hernández, M. (s.f.). El niño yun- } \\
\text { tero. https://www.poemas-del- } \\
\text { alma.com/miguel-hernandez-el- } \\
\quad \text { nino-yuntero.htm }\end{array}$ & $\begin{array}{l}\text { Lectura en voz alta colectiva de los poemas seleccio- } \\
\text { nados. La Act.1 consistió en escribir un texto creativo } \\
\text { que se relacionara con algún oficio u actividad que les } \\
\text { pareciera interesante. Para continuar, se realizaron } \\
\text { comentarios acerca de la lectura; finalmente, se repro- } \\
\text { dujo la canción El niño yuntero }\end{array}$ \\
\hline $\begin{array}{l}\text { Sesión } 15 \\
\text { Asistentes: } 16 \\
\text { Fecha: } \\
\text { 27/02/2020 }\end{array}$ & $\begin{array}{l}\text { Presentación del documental de } \\
\qquad 90 \text { minutos: } \\
\text { Schwarz, S. (director) (2012). } \\
\text { Narco cultura. Coproducción } \\
\text { EUA-México }\end{array}$ & $\begin{array}{l}\text { Presentación del documental } \\
\text { (proyección de } 45 \text { minutos) }\end{array}$ \\
\hline $\begin{array}{l}\text { Sesión } 16 \\
\text { Asistentes: } 17 \\
\text { Fecha: } \\
\text { 04/03/2020 }\end{array}$ & $\begin{array}{l}\text { Presentación del documental de } \\
90 \text { minutos: Schwarz, S. (di- } \\
\text { rector) (2012). Narco cultura. } \\
\text { Coproducción EUA-México }\end{array}$ & $\begin{array}{l}\text { Proyección de } 45 \text { minutos. Apertura de mesa de diálo- } \\
\text { go acerca de los puntos de vista, reflexiones y comen- } \\
\text { tarios surgidos a partir del contenido. Se identificaron } \\
\text { algunos tipos de violencia. Se habló sobre la cultura de } \\
\text { la violencia y su naturalización }\end{array}$ \\
\hline
\end{tabular}




\begin{tabular}{|c|c|c|}
\hline $\begin{array}{l}\text { Sesión } 17 \\
\text { Asistentes: } 17 \\
\text { Fecha: } \\
\text { 10/03/2020 }\end{array}$ & $\begin{array}{l}\text { Keret, E. (2016). Romper el cerdi- } \\
\text { to. Sexto Piso } \\
\text { Satie, E. (1974). Three Gymnopé- } \\
\text { dies I: Lent et douloureux [álbum]. } \\
\text { Amazon music (trabajo original } \\
\text { publicado en 1888) }\end{array}$ & $\begin{array}{l}\text { Lectura en voz alta individual completa del cuento de } \\
\text { Edgar Keret. Se inició el Taller de escritura creativa II, } \\
\text { se repartieron hojas blancas y se les pidió que escri- } \\
\text { bieran un cuento de tema abierto; mientras tanto, se } \\
\text { reprodujo la pieza musical de Erik Satie. Se les propor- } \\
\text { cionó } 40 \text { minutos para terminar la actividad }\end{array}$ \\
\hline $\begin{array}{l}\text { Sesión } 18 \\
\text { Asistentes: } 17 \\
\text { Fecha: } \\
\text { 11/03/2020 }\end{array}$ & $\begin{array}{l}\text { Crosthwaite, L. H. (2009). Aparta } \\
\text { de mí este cáliz. Tusquets (pp. 11- } \\
14 \text { y pp. 23-26) } \\
\text { Monterroso, A. (1994). La oveja } \\
\text { negra y demás fábulas. Ediciones } \\
\text { Era }\end{array}$ & $\begin{array}{l}\text { Lectura en voz alta colectiva de los fragmentos selec- } \\
\text { cionados de Crosthwaite. Lectura en voz alta individual } \\
\text { de las fábulas de Monterroso. Posteriormente, se aplicó } \\
\text { la encuesta final y se emitió un discurso sobre el cierre } \\
\text { del taller, agradecimientos y despedida con el grupo }\end{array}$ \\
\hline
\end{tabular}

\section{RESULTADOS}

El $67 \%$ de los participantes asistieron al $75 \%$ de las sesiones y el $50 \%$ a todas las sesiones; el $21 \%$ a la mitad o menos. Se registró una alta participación: 69\% estuvieron presentes en las actividades propuestas. Los resultados, de acuerdo con los objetivos planteados, se detallan a continuación.

Suscitar en los jóvenes el interés por la lectura y la escritura a través de la lectura estética y la escritura creativa

En la encuesta diagnóstica, el 75\% de los participantes (18 de 24) afirmaron que no les gustaba leer. No obstante, al preguntar si tenían el hábito lector, contestaron lo que mostramos en la gráfica 1 . Menos de la mitad ejercitaba la lectura y quienes respondieron que tenían el hábito leían máximo dos horas a la semana, lo cual evidencia su desconocimiento sobre el significado de hábito lector. El 29\% que respondió que no le gustaba leer, al finalizar la intervención uno reafirmó su disgusto por la lectura, dos dejaron de asistir y tres modificaron su respuesta a "les gusta leer".

Gráfica 1. Hábitos de lectura

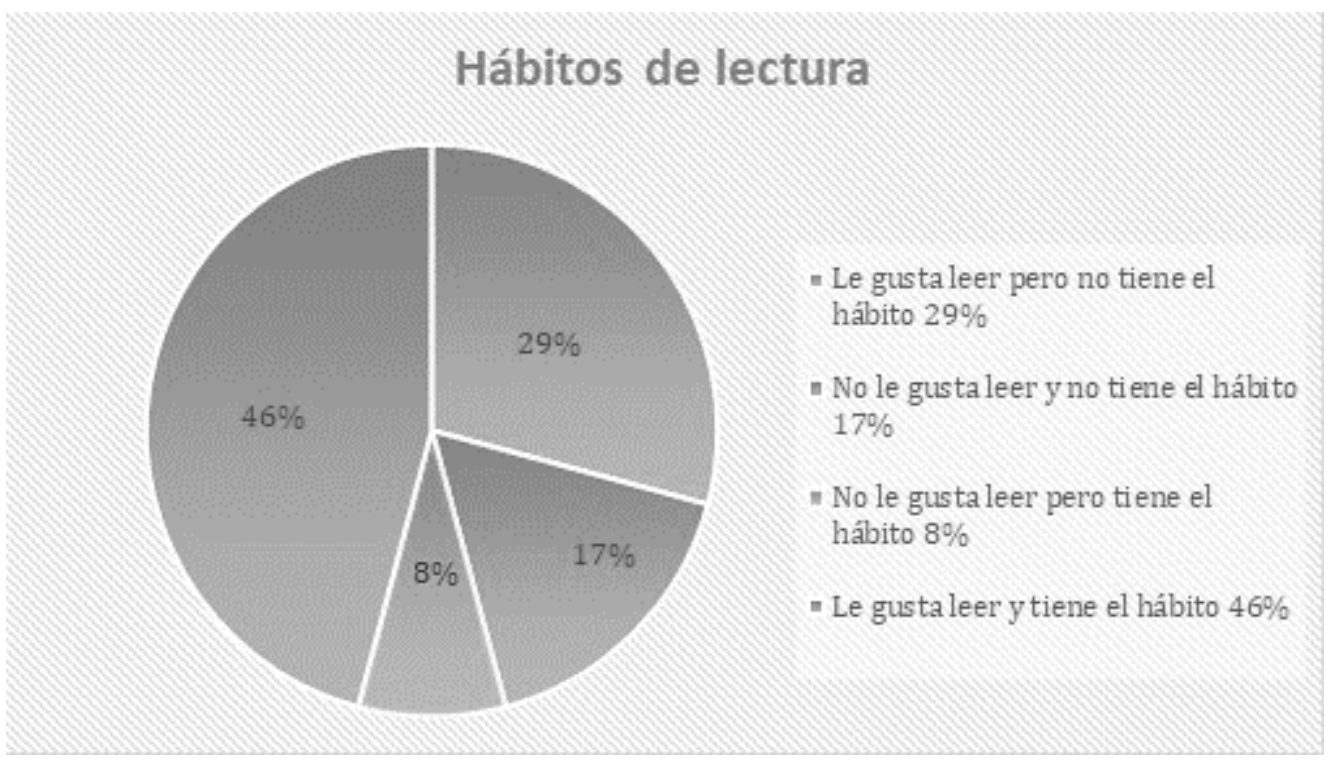

Lara / Jarvio. Marginación educativa y violencia: una intervención de lectoescritura en telebachillerato Sinéctica 57 www.sinectica.iteso. $m x$ 
Al preguntar si continuarían leyendo después de concluir la intervención, 14 de 17 dijeron que sí; 11 de 17 respondieron que cambió el tiempo semanal de lectura, de los cuales 9 aseguraron haber leído en casa: revistas, historietas, novelas, textos informativos, cuentos y libros como El diario de Greg, La ladrona de libros, Enciclopedia de animales, Las batallas en el desierto y La estrella de la quinta avenida. Los títulos más exitosos fueron: El fútbol a sol y sombra, Las batallas en el desierto, Romper el cerdito, El amor, las mujeres, El país de las metrallas, y el favorito: Estrella de la calle sexta. El más desdeñado fue La gallina degollada.

En relación con el desempeño en la escritura creativa, aumentó el interés y dedicación especialmente en las sesiones de "Escribiendo cuentos I y II". En la primera, el 70\% (17 de 24) apenas logró escribir menos de media cuartilla, mientras que, en la segunda, redactaron de una cuartilla y media a tres. En "Escribiendo cuentos I", los jóvenes elaboraron relatos basados en anécdotas personales, en tanto que en "Escribiendo cuentos II" aprovecharon recursos y estructuras literarias correspondientes al cuento -introducción, nudo y desenlace-; realizaron descripciones de personajes y lugares; utilizaron frases como "en tierras lejanas", "había/érase una vez" y emplearon diferentes registros para los personajes. De esta forma, emplearon sus conocimientos previos, creatividad e imaginación, y consideraron no solo la diégesis, sino que concientizaron el tipo de discurso a ejecutar.

Acercar a los jóvenes a las manifestaciones artísticas relacionadas con la lectura y la escritura

No hubo condiciones para el uso de recursos multimedia. No obstante, se presentaron temas musicales y el documental. Los jóvenes se mantuvieron atentos e interesados al descubrir los vínculos que existen entre la literatura y otras manifestaciones culturales, como la música, y pudieron relacionar las temáticas con los textos leídos.

Propiciar un cambio de actitudes y prejuicios relacionados con el estereotipo del lector

En la encuesta diagnóstica se muestra que no existía una idea clara sobre lo que significa ser lector. En su mayoría, se expresaron conceptos que tienen que ver con el significado utilitario de la lectura: "para aprender, ser sabio o un estudiante aplicado"; le siguieron los que respondieron "no sé" o no contestaron. Sin embargo, en la encuesta final, observamos una mejor opinión de lo que es un lector, ya que integraron conceptos como la imaginación, la creatividad y los sentidos, lo que se relaciona con la lectura estética.

Incentivar la expresión, reflexión, diálogo para desnaturalizar y comprender el fenómeno de la violencia

El discurso sobre la violencia estuvo presente en siete sesiones en textos de autores como Felipe Rodríguez, Horacio Castellanos Moya, entre otros, con la finalidad de desnaturalizar y comprender la violencia mediante la expresión, la reflexión y el diálogo. En dos de las sesiones implementamos la reflexión escrita; en las siete, el diálogo y la reflexión oral; y en cuatro se identificaron los tipos de violencia y las 
ideologías en los discursos: 22 de 24 jóvenes consideraron importante hablar sobre este tema, y expresaron sus razones (ver gráfica 2).

Gráfica 2. La importancia de dialogar y reflexionar sobre la violencia

\section{¿Por qué es importante dialogar o reflexionar acerca de la violencia?}

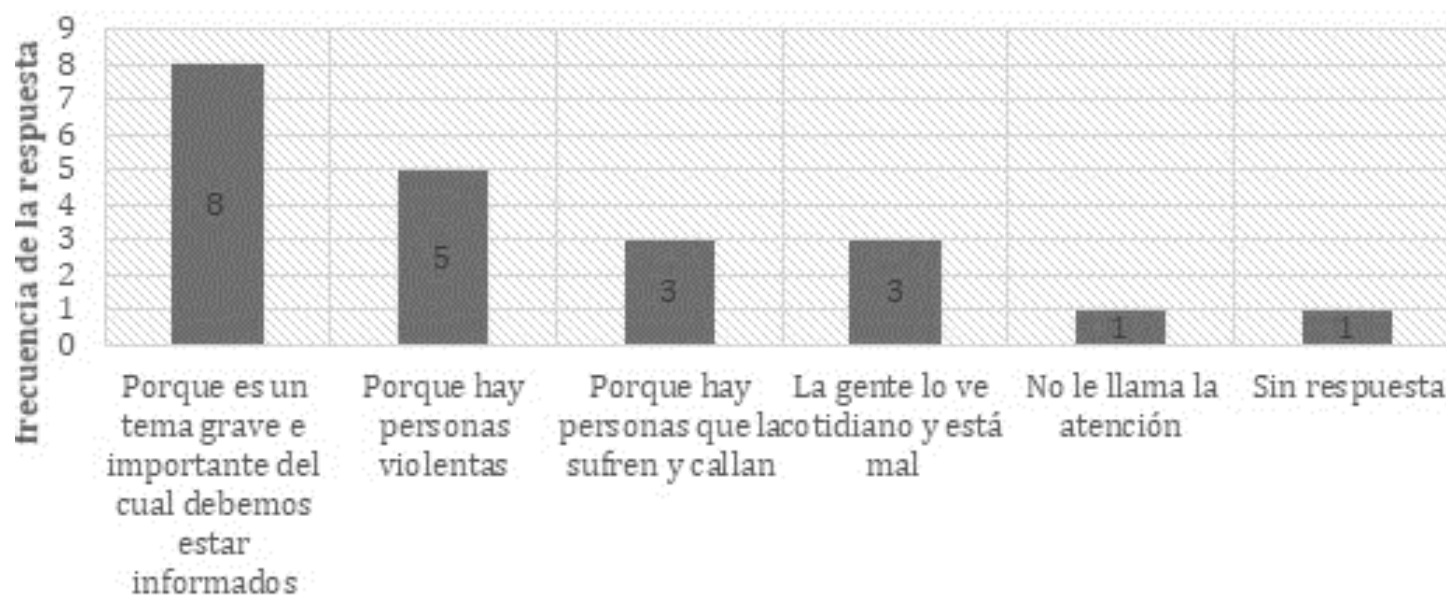

Respuestas

De 17 participantes, 14 afirmaron que cambió su perspectiva en torno al tema. La tabla 2 contiene sus respuestas.

Tabla 2. Reflexiones textuales sobre la violencia

\begin{tabular}{|c|c|}
\hline $\begin{array}{c}\text { Reflexión sobre la pregunta ¿cambió la percepción } \\
\text { que tenías acerca de la violencia? ¿Por qué? }\end{array}$ & $\begin{array}{c}\text { Reflexión sobre la pregunta ¿consideras que el ta- } \\
\text { ller te ayudó a reflexionar, dialogar, leer y escribir } \\
\text { acerca de la violencia? }\end{array}$ \\
\hline $\begin{array}{c}\text { Sí, porque ambos nos podemos hacer daño } \\
\text { dí, ahora las cosas están más tranquilas, hay tipos } \\
\text { de ella }\end{array}$ & Eude ampliar mi conocimiento hay muchos tipos de violencia \\
\hline $\begin{array}{c}\text { Sí, porque lo que pensaba no era en realidad, } \\
\text { bueno sí, pero no muy bien }\end{array}$ & Pude entender más sobre ella y los tipos \\
\hline $\begin{array}{c}\text { Sí, ahora estoy en contra y entiendo un poco más } \\
\text { de eso }\end{array}$ & Otra forma de ver las cosas, los tipos \\
\hline Para que eso casi no exista & Te vas dando cuenta que las cosas son malas \\
\hline
\end{tabular}




\begin{tabular}{|c|c|}
\hline Conocí los tipos de violencia & $\begin{array}{l}\text { Pues reflexionamos y pensar qué es la violencia, } \\
\text { cuánta violencia hay, qué puedo hacer para que se } \\
\text { acabe }\end{array}$ \\
\hline Sí, los daños que podría causar & Relacionar y solucionar el problema \\
\hline & De manera recreativa y me ayuda a sacar el dolor \\
\hline & De manera positiva \\
\hline & Aprendo nuevas palabras \\
\hline & Porque yo la veo como una manera más seria \\
\hline & $\begin{array}{l}\text { Aprendí más palabras y tenía mejor para poder } \\
\text { hablar }\end{array}$ \\
\hline & Pensamiento \\
\hline
\end{tabular}

Finalmente, en este ámbito, observamos que las reflexiones sobre la violencia están diferenciadas por género; es decir, las mujeres pensaron con mayor detenimiento el tema de la violencia familiar y la que se ejerce contra la mujer, mientras que los hombres hablaron sobre violencia económica y laboral.

Tabla 3. Reflexiones textuales en torno a la violencia según género

\begin{tabular}{|c|c|}
\hline Mujeres & Hombres \\
\hline $\begin{array}{l}\text { 1. A veces, la violencia puede ser el golpe más } \\
\text { duro en la vida, más cuando son de la familia; la } \\
\text { violencia en estos tiempos ha arrasado el mun- } \\
\text { do entero: del } 70 \% \text { de la violencia de padres } \\
\text { a hijos menores vienen reproches entre ellos, } \\
\text { discusiones, etc.; es mejor un hijo perdido a un } \\
\text { hijo deprimido, por eso luchemos a legalizar el } \\
\text { aborto para que no vengan a sufrir los hijos }\end{array}$ & $\begin{array}{l}\text { 1. La violencia es un término y acción horrible } \\
\text { que se vive en las calles, trabajos, etc., esa horrible } \\
\text { acción nunca se ha podido acabar gracias a que } \\
\text { gobiernos y sociedades la han permitido y quien } \\
\text { no las ha aceptado las ha tenido que vivir; primero } \\
\text { se sabe en familias ahora se da demasiado en los } \\
\text { trabajos gracias a que hay gente débil y temerosa } \\
\text { que nunca habla y siempre se traga la verdad }\end{array}$ \\
\hline $\begin{array}{l}\text { 2. En el país, la mayoría de la violencia es contra } \\
\text { las mujeres, ya que algunos dicen que las muje- } \\
\text { res solo venimos al mundo a servir a los demás } \\
\text { y eso no es cierto, tenemos voz y eso no debe } \\
\text { ser así, que la violencia se acabe, todos tenemos } \\
\text { que pensar a quien afectamos cuando hablamos }\end{array}$ & $\begin{array}{l}\text { 2. La violencia no puede ser justificada, pero en } \\
\text { algunos casos las personas pueden llegar a tener } \\
\text { cierto grado de complicidad: los gobiernos mexi- } \\
\text { canos al dejarlos sin un apoyo laboral seguro }\end{array}$ \\
\hline
\end{tabular}

Suscitar el interés por la lectura y la escritura mediante la lectura estética, la escritura creativa y otras manifestaciones artísticas propicia un cambio de actitudes y prejuicios relacionados con el estereotipo de ser lector. La lectura debe ser adoptada como una actividad cotidiana, personal y enriquecedora, que puede incentivar la 
expresión, la reflexión y el diálogo para desnaturalizar y comprender el fenómeno de la violencia.

\section{DiSCUSIÓN Y CONCLUSIONES}

El diseño del trabajo utilizó las experiencias de otros proyectos tanto nacionales como internacionales. La característica común fue la de establecer programas que sensibilizaran sobre el tema de la violencia para dejar de normalizar prácticas como el acoso, la discriminación y la violencia física, verbal o psicológica. Aunque en su mayoría no eran proyectos para fomentar la lectura, todos utilizaban textos literarios para reflexionar sobre el tema y, así, fomentar el diálogo y la reflexión crítica para promover una cultura de paz, como lo mencionan Volpi (2011) y Yubero, Sánchez y Larrañaga (2013).

La cartografía lectora que usamos se adecuó a los intereses del grupo; destaca que los textos sobre violencia causaron más atracción que los de literatura universal, lo que permitió abordar el tema de forma natural y entusiasta. Los datos reportados se obtuvieron a través de las bitácoras empleadas en cada sesión y en ellas se anotaban comentarios, participaciones y razonamientos significativos. En cada sesión se propiciaron escenarios diferentes a las actividades cotidianas en el salón de clases. Observamos que valoraban mucho la relación horizontal entre la responsable del proyecto y los estudiantes, lo que originó un acercamiento igualitario en el intercambio de experiencias, así como una aproximación atenta a su contexto. Ello promovió el involucramiento de los estudiantes para participar en todas las actividades y lograr, como lo establece la metodología de investigación-acción participativa, ciertas transformaciones del entorno (Callejo y Viedma, 2006; Sigalat et al., 2019).

La vinculación con los profesores se circunscribió a la realización de gestiones administrativas para la operación del proyecto de intervención, así como para obtener los avales para su implementación, en los que cuidamos el manejo ético en cada actividad. Los docentes manifestaron poco interés en involucrarse en las actividades con el argumento de ser pocos y tener que atender muchas labores. No obstante, insistimos en la importancia de crear una biblioteca y fomentar la lectura en virtud de los resultados obtenidos.

Como ejemplo de lo anterior, se reporta que hubo un aumento en el interés por leer que se evidencia en el tiempo dedicado a la lectura en casa, además de que siete de los asistentes señalaron que compartieron las lecturas con familiares cercanos como padres o hermanos, situación que amplía el impacto de acercamiento a prácticas de lectura y que abre un área de oportunidad para intervenciones posteriores.

Como lo indican Garbe (2005) y Garrido (2012), es fundamental la motivación en esta etapa de la vida, así como de los contextos de apoyo que amplían las posibilidades de acercamiento a los libros. Ante la falta de una biblioteca en la escuela, los estudiantes solicitaron a la instructora los libros utilizados para ser leídos en casa. La lectura en voz alta fue una herramienta esencial de gran aceptación en el grupo, ya que, como destaca Garrido (2004), “cuando alguien [...] lee para niños o para adultos con emoción, interés y cuidado, se crea un espacio de complicidad privilegiado" (p. 67). 
En relación con la escritura creativa, observamos avances dado el esfuerzo realizado, así como muestras de imaginación y creatividad que se evidencian en los escritos producto de un permanente diálogo en la percepción de un mismo fenómeno (Barrón, 2019); también, distinguimos otras formas expresivas como el dibujo.

$\mathrm{Al}$ experimentar la lectura como un acto disfrutable, que además permite el conocimiento del entorno, cambió la idea de lo que inicialmente significaba ser lector. Al final del taller, más del $80 \%$ asoció el ser lector con elementos como la imaginación, la creatividad, los sentimientos, la astucia, la habilidad y el gusto, y destacó la importancia de la dedicación permanente a esta actividad.

Las actividades desarrolladas en cada sesión propiciaron el sentido y el significado de los textos; se incentivó la expresión, la reflexión y el diálogo que permitieron comprender el fenómeno de la violencia y eliminar, así, el significado de "acción natural". Las lecturas compartidas tienen como particularidad formas transgresoras con hechos ciertos que se abordan desde la lectura estética (Morales, 2019). Alternar un texto sobre violencia por cada dos de literatura universal fue un acierto. Resalta, además, las diferencias de opinión por género, aunque al final existió unanimidad al expresar tener una mejor comprensión sobre el fenómeno y, ante todo, a decir "no a la violencia".

No obstante, todas las actividades realizadas tuvieron múltiples contratiempos y limitantes debido a que la institución educativa no cumple con las condiciones básicas para el desarrollo de las actividades académicas, lo que da lugar a la prevalencia de grandes brechas (Weiss et al., 2017).

La primera gran limitante fue la inconsistencia del tiempo entre sesión y sesión. En el periodo de la intervención se incumplió de manera sistemática el calendario escolar, y se dedicó tiempo a diversas actividades no siempre relacionadas con el plan de estudios. Esto pudo alterar los resultados del proyecto, ya que, como establece Bangert-Drowns (2004, citado en Hernández-Espíndola 2016), se ha determinado que el impulso de tareas frecuentes (dos o tres veces por semana) y cortas durante un periodo largo tienen más efectos positivos que las tareas largas, infrecuentes y por lapsos más cortos.

Otras actividades se cancelaron por falta de permisos por parte de la institución, como la invitación a autores de libros y booktubers. Tampoco hubo condiciones para el empleo de recursos multimedia ni para poner en marcha el plan completo elaborado para acercar a los jóvenes a diversas manifestaciones artísticas relacionadas con la literatura. Este objetivo fue el que se cumplió en menor medida.

Por lo anterior, es posible justificar el abandono escolar en el telebachillerato. En México, el promedio de la tasa de eficiencia terminal en la EMS es del $64.4 \%$ y el indicador más alto le corresponde al estado de Veracruz, con el $80.1 \%$ (Instituto Nacional para la Evaluación de la Educación, 2019); no obstante, en este centro, solo en el tiempo de la intervención, hubo un abandono escolar de siete estudiantes de un total de 24, en su mayoría de sexo masculino.

Finalmente, es importante señalar que los resultados de los objetivos planteados se cumplieron de modo satisfactorio a pesar de los inconvenientes, lo que demuestra que un trabajo permanente y sistemático de promoción de lectura en jóvenes es viable y podría coadyuvar a la construcción de una educación de calidad y una sociedad con menor arraigo a la violencia. 


\section{REFERENCIAS BIBLIOGRÁFICAS}

Aguilar-Caro, A. I. (2015). Incidencia de fomento lector en la disminución de violencia en el caribe colombiano. En ACLEBIM. Asociación de profesionales de bibliotecas móviles (pp. 1-10). https://bibliobuses.com/wp-content/ uploads/documentos/Aura\%20Aguilar\%202015.pdf

Barrón Tirado, M. C. (2019). De la artesanía académica al goce artístico. En E. M. Ramírez Leiva (coord.). De la lectura académica a la lectura estética (pp. 49-58). México: UNAM.

Callejo Gallego, M. J. y Viedma Rojas, A. (2006). Proyectos y estrategias de investigación social: la perspectiva de la intervención. España: McGraw-Hill.

Cassany, D. (2006). Tras las líneas. Barcelona: Anagrama.

Fundación Mujeres (2017). Tipos de violencia contra las mujeres y prevención de violencia en adolescentes. http://www.fundacionmujeres.es/img/Document/67259/documento.pdf

Gabbian B. y Orlando V. (2018). Los textos académicos como géneros discursivos. En V. Bertolotti (comp.). Lengua, comunicación e información. Montevideo: Facultad de Información y Comunicación. http://www.fic.edu.uy/sites/ default/files/201902/lengua_comunicacion_informacion_CC.pdf

Garbe, C. (2005). Investigación de la lectura en Alemania. Métodos y resultados. En E. M. Ramírez Leyva (ed.). Lectura: pasado, presente y futuro. México: UNAM.

García García, M. A., Arévalo Duarte, M. A. y Hernández Suárez, C. A. (2018). La comprensión lectora y el rendimiento escolar. Cuadernos de Lingüística Hispánica, vol. 32, pp. 1-25.

Garrido, F. (2012). Manual del buen promotor. Una guía para promover la lectura y la escritura. México: Alas y raíces/Conaculta.

Garrido, F. (2004). Para leerte mejor. Mecanismos de la lectura y de la formación de lectores. México: Planeta.

Garrido, F. (1998). Cómo leer mejor en voz alta. México: SEP.

Grijalva Martínez, O. (2011). La diversión y el trabajo académico como fuentes de las identificaciones de los jóvenes en sus grupos de pares. Revista de Investigación Educativa, núm. 12. https://www.uv.mx/cpue/num12/inves/ completos/grijalva-diversion-trabajo-academico.html

Guthrie, J. T., Wigfield, A., \& Perencevich, K. C. (Ed.). (2004). Motivating reading comprehension: Concept-oriented reading instruction. Mahwah, NJ: Erlbaum.

Guthrie, J. T., Wigfield, A., Humenick, N. M., Perencevich, K. C., Taboada, A. \& Barbosa, P. (2006). Influences of stimulating tasks on reading motivation and comprehension. Journal of Educational Research, núm. 99, pp. 232-245.

Hernández-Espíndola, D. (2016). Escribir desde la perspectiva del género textual: el desarrollo cognitivo a escena. En D. Hernández-Espíndola. Escritura y desarrollo cognitivo en un mundo intertextual: diálogos con la obra de Charles Bazerman (pp. 59-85). México: BUAP.

Instituto de la Juventud (2009). Talleres de prevención de violencia de género. http://www.injuve.es/sites/default/files/foroviolenciagenero-talleres.pdf

Institute for Economics \& Peace (2018). Índice de paz México. http://indicedepazmexico.org/wp-content/uploads/2018/04/Indice-de-Paz-Mexico-2018.pdf

Instituto Nacional parala Evaluación dela Educación(2019). La educación obligatoria en México. https://www.inee.edu.mx/medios/informe2019/stage_01/ 
cap_0103.html\#: :text=Ambas\%20entidades\%20tambi\%C3\%A9n\%20tienen $\% 20$ los, $20.6 \% 25 \% 20$ (tabla\%201.6)

Instituto Nacional para la Evaluación de la Educación (2017b). Evaluación de la oferta educativa (EVOE) en EMS. Condiciones escolares e implementación curricular. https://www.inee.edu.mx/wp-content/uploads/2019/06/P2A333.pdf

Instituto Nacional para la Evaluación de la Educación (2017a). PLANEA Resultados nacionales 2017. México. http://planea.sep.gob.mx/content/general/ docs/2017/ResultadosNacionalesPlaneaMS2017.PDF

Jacinto-Roca, L. E., Landa Galarza, R. R. y López Rodríguez, C. R. (2018). La lectura en voz alta como estrategia metodológica para mejorar la comprensión lectora en estudiantes del $3^{\circ}$ grado de educación primaria de la IE José Olaya Balandra 6090-Chorrillos. http://200.60.81.165/handle/UNE/1928

López, A. (2017). La violencia de género en el aula de literatura. Un proyecto por la sensibilización con la violencia de género en el aula de literatura a través de cuentos de Emilia Pardo Bazán. Didáctica. Lengua y literatura, núm. 29, pp. 235-258. file:///C:/Users/Lenovo/Downloads/57141-Texto\%20del\%20 art\%C3\%ADculo- 114705-2-10-20171221.pdf

Loría-Canul, D. P. (2018). Caravana literaria: creando inquietudes lectoras y cambiando realidades sociales. En Secretaría de Cultura. Premio para el fomento de la escritura y la lectura 2017 (pp. 41-51). México: Secretaría de Cultura. https://pro.librosmexico.mx/adjuntos/772119-1-81594.PDF

Martínez Miguélez, M. (2004). Ciencia y arte en la metodología cualitativa. México: Trillas.

Montealegre Aguilar, A. (2011). Leer y escribir para investigar en la educación superior: un proceso. Actualidades Pedagógicas, núm. 57, pp. 199-212. https:// ciencia.lasalle.edu.co/cgi/viewcontent.cgi?article=1033\&context=ap

Morales Sánchez, M. I. (2019). La lógica de la lectura: de la lectura estética o "el arte de leer con sentido". En E. Ramírez Leiva (coord.). De la lectura académica a la lectura estética (pp. 59-76). México: UNAM.

Observatorio de la Juventud en Iberoamérica (2019). Encuesta de Jóvenes en México 2019. https://drive.google.com/file/d/1QNRuGhuSMSOV3Ky2fAPH o6otNtFORskk/view

Organización para la Cooperación y el Desarrollo Económicos (OCDE) (2018). Programa para la Evaluación Internacional de Alumnos (PISA). PISA 2018. https://www.oecd.org/pisa/publications/PISA2018_CN_MEX_Spanish.pdf

Organización para la Cooperación y el Desarrollo Económicos (OCDE) (2006). El programa PISA de la OCDE. Qué es y para qué sirve. https://www.oecd.org/ pisa/39730818.pdf

Pérez, I. G. (2018). Leer en la universidad. En L. Natale y D. Stagnaro. La lectura y la escritura en las disciplinas. Lineamientos para su enseñanza (pp. 59-112). Buenos Aires, Argentina: Universidad Nacional de General Sarmiento.

Pirela Morillo, J. (2019). Lectura estética como estrategia transversal para la formación académica de profesionales universitarios. En E. Ramírez Leiva (coord.). La lectura académica a la lectura estética (pp. 35-48). México: UNAM.

Pontecorvo, C. (2002). Las prácticas de alfabetización escolar: ¿es aún válido el hablar bien para escribir bien? En E. Ferreiro (comp.). Relaciones de (in)dependencia entre oralidad y escritura (pp. 133-149). Barcelona, España: Gedisa Editorial. 
Ramírez Leyva, E. M. (coord.) (2019). De la lectura académica a la lectura estética. México: UNAM. http://ru.iibi.unam.mx/jspui/bitstream/IIBI_UNAM/ L217/1/L222.pdf

Rosen, J. D. y Zepeda Martínez, R. (2015). La guerra contra el narcotráfico en México: una guerra perdida. Reflexiones, núm. 1, pp. 153-168. https://www.scielo. sa.cr/pdf/reflexiones/v94n1/1659-2859-reflexiones-94-01-00153.pdf

Secretaría de Desarrollo Social (Sedesol) (2014). Programa Nacional de Juventud 2014-2018. http://politicasdejuventud.celaju.net/wp-content/ uploads/2014/05/projuventud-2014-2018-documento-base1.pdf

Secretaría de Desarrollo Social (Sedesol) (2010). Catálogo de localidades http://www.microrregiones.gob.mx/catloc/LocdeMun.aspx?tipo=clave\&ca mpo=loc\&ent $=30 \&$ mun $=038$

Secretariado Ejecutivo del Sistema Nacional de Seguridad Pública (2020). Informe de incidencia delictiva. https://drive.google.com/file/ d/1D0U76MBPHLEW06k78XIWFm1-PC3PVW1B/view

Segato, R. L. (2003). Las estructuras elementales de la violencia. Argentina: Bernal, Argentina: Universidad Nacional de Quilmes. http://mercosursocialsolidario.org/valijapedagogica/archivos/hc/1-aportes-teoricos/2.marcos-teoricos/3.libros/RitaSegato.LasEstructurasElementalesDeLaViolencia.pdf

Sigalat Signes, E., Calvo Palomares, R., Roig Merino, B. y Buitrago Vera, J. M. (2019). Investigación acción participativa (IAP) en el sector empresarial. Interviniendo desde lo local. EMP1RIA. Revista de Metodología de Ciencias Sociales, núm. 44, pp. 47-78.

Valenzuela, J. M. (2017). Juventudes demediadas. Desigualdad, violencia y criminalización de los jóvenes en México. En R. Reguillo (coord.). Los jóvenes en México (pp. 271-299). México: Fondo de Cultura Económica.

Vázquez Ahumada, A. (2003). El prodigio de la escritura. En M. T. F. Encinas Prudencio (coord.). Escritura y desarrollo cognitivo en un mundo intertextual (pp. 39-55). México: BUAP.

Volpi, J. (2011). Leer la mente. El cerebro y el arte de la ficción. México: Alfaguara.

Weiss, E. (2018). Los significados del bachillerato para los jóvenes y la permanencia escolar en México. Sinéctica, Revista Electrónica de Educación, núm. 51, pp. 1-19. https://sinectica.iteso.mx/index.php/SINECTICA/article/ view/856/1020

Weiss, E. (2012). Jóvenes y bachillerato. México: Asociación Nacional de Universidades e Instituciones de Educación Superior. http://departamentos. cinvestav.mx/Portals/die/SiteDocs/Investigadores/EWeiss/Jovenes/EWJovenes2012-Jovenes_y_bachillerato.pdf\#page=7

Weiss, E., Antonio, L., Bernal, E., Guzmán, C. y Pedroza, P. (2017). El telebachillerato comunitario. Una innovación curricular a discusión. Revista Latinoamericana de Estudios Educativos, vol. 47, núm. 3, pp. 7-26. http://cee.edu.mx/ home/la-agendaeducativa-3/

Wigfield, A. \& Wagner, A. L. (2005). Competence, motivation, and identity development during adolescence. En A. J. Elliot \& C. S. Dweck (eds.). Handbook of competence and motivation (pp. 222-239). Nueva York: Guilford Publications.

Yubero Jiménez, S., Sánchez García, S. y Larrañaga, E. (2013). Valores y lectura. En E. Martos Núñez y M. C. Fernández-Figares (coords.). Diccionario de nuevas formas de lectura y escritura (pp. 663-666). Madrid: Red Internacional de Universidades Lectoras/Santillana. 